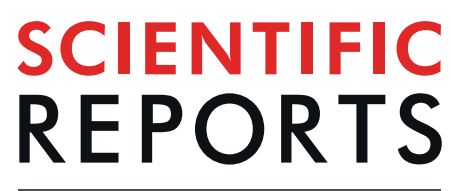

natureresearch

\title{
OPEN Network Systems Pharmacology- Based Mechanism Study on the Beneficial Effects of Vitamin D against Psychosis in Alzheimer's Disease
}

\begin{abstract}
Peihao Fan ${ }^{1}$, Xiguang $\mathrm{Oi}^{1}$, Robert A. Sweet ${ }^{2,3^{*}}$ \& Lirong Wang ${ }^{1 *}$
Alzheimer's disease (AD) is a chronic neurodegenerative disease with significant financial costs and negative impacts on quality of life. Psychotic symptoms, i.e., the presence of delusions and/ or hallucinations, is a frequent complication of $A D$. About $50 \%$ of $A D$ patients will develop psychotic symptoms ( $A D$ with $P$ sychosis, or $A D+P$ ) and these patients will experience an even more rapid cognitive decline than $A D$ patients without psychosis (AD-P). In a previous analysis on medication records of $776 \mathrm{AD}$ patients, we had shown that use of Vitamin $D$ was associated with delayed time to psychosis in $A D$ patients and Vitamin $D$ was used more by $A D-P$ than $A D+P$ patients. To explore the potential molecular mechanism behind our findings, we applied systems pharmacology approaches to investigate the crosstalk between $A D$ and psychosis. Specifically, we built protein-protein interaction (PPI) networks with proteins encoded by AD- and psychosis-related genes and Vitamin D-perturbed genes. Using network analysis we identified several high-impact genes, including NOTCH4, COMT, CACNA1C and DRD3 which are related to calcium homeostasis. The new findings highlight the key role of calcium-related signaling pathways in $A D+P$ development and may provide a new direction and facilitate hypothesis generation for future drug development.
\end{abstract}

Alzheimer's disease (AD) is a chronic neurodegenerative disease commonly seen in the aging population, and the presence of $\mathrm{AD}$ is responsible for a significant decrease in the quality of life ${ }^{1}$. It is estimated that the cost of AD is $\$ 604$ billion worldwide and will triple in Year $2050^{2}$. Genetic factors are the second strongest risk factor for AD following age $\mathrm{e}^{3}$. However, multiple environmental factors can be associated with the development of AD including medication usage ${ }^{4}$.

Psychosis, defined by the occurrence of delusions and/or hallucinations, is observed as a common complication of $\mathrm{AD}$ and patients with dementia. Literature reports that approximately $50 \%$ of AD patients will have psychotic symptoms ( $\mathrm{AD}$ with psychosis, or $\mathrm{AD}+\mathrm{P}$ ) in the following years ${ }^{5}$. $\mathrm{AD}+\mathrm{P}$ patients are considered as a subgroup of patients who have more severe symptoms, and are associated with more significant cognitive impairment and a quicker cognitive decline ${ }^{6} . \mathrm{AD}+\mathrm{P}$ is also associated with higher rates of co-occurring agitation $^{7}$, aggression ${ }^{7,8}$, depression ${ }^{9,10}$, mortality ${ }^{11}$, functional impairment ${ }^{12}$, and increased caregiver burden ${ }^{13}$ than AD patients without psychosis (AD-P).

Psychosis in $\mathrm{AD}$ often represents a significant additional drain on top of the baseline disease burden. $\mathrm{AD}+\mathrm{P}$ patients are found to have a more rapid decline in the cognitive and memory functions, and the presence of psychosis can significantly increase the difficulty for caregivers. The symptoms of psychosis can cause considerable distress to patients ${ }^{14-16}$. In addition to these effects, $\mathrm{AD}+\mathrm{P}$ is a marker for other adverse outcomes in $\mathrm{AD}$. The most associated behavior disturbances with $\mathrm{AD}+\mathrm{P}$ are agitation and aggression; ${ }^{7,8,17}$ depressive symptoms are also increased in $\mathrm{AD}+\mathrm{P}$ patients ${ }^{9,10}$.

\footnotetext{
${ }^{1}$ Department of Pharmaceutical Sciences, Computational Chemical Genomics Screening Center, University of Pittsburgh School of Pharmacy, Pittsburgh, USA. ${ }^{2}$ Department of Neurology, University of Pittsburgh School of Medicine, Pittsburgh, USA. ${ }^{3}$ Department of Psychiatry, University of Pittsburgh School of Medicine, Pittsburgh, USA. *email: sweetra@upmc.edu; liw30@pitt.edu
} 


\begin{tabular}{|l|l|l|l|l|}
\hline Network Name & $\begin{array}{l}\text { Node } \\
\text { Number }\end{array}$ & $\begin{array}{l}\text { Edge } \\
\text { Number }\end{array}$ & $\begin{array}{l}\text { Average Degree } \\
\text { Centrality }\end{array}$ & $\begin{array}{l}\text { Average Betweenness } \\
\text { Centrality }\end{array}$ \\
\hline Antipsychotics & 89 & 419 & 0.106 & 0.0157 \\
\hline Psychosis & 486 & 1409 & 0.0119 & 0.00642 \\
\hline $\begin{array}{l}\text { Psychosis-antipsychotics } \\
\text { Combined Network }\end{array}$ & 570 & 1825 & 0.0112 & 0.00563 \\
\hline
\end{tabular}

Table 1. Characteristics of Antipsychotics- and Psychosis-related PPI networks.

\begin{tabular}{|l|l|l|}
\hline Gene Name & Degree Centrality & Betweenness Centrality \\
\hline DRD2 & 0.0703 & 0.1433 \\
\hline HTR2A & 0.058 & 0.0731 \\
\hline GRIA1 & 0.0615 & 0.0698 \\
\hline ALB & 0.0633 & 0.0677 \\
\hline CACNA1C & 0.0545 & 0.0513 \\
\hline FOS & 0.0615 & 0.05 \\
\hline SYNE1 & 0.0246 & 0.0488 \\
\hline GRIN2A & 0.0545 & 0.0485 \\
\hline FYN & 0.0545 & 0.0432 \\
\hline KIT & 0.0422 & 0.0368 \\
\hline
\end{tabular}

Table 2. Overview of net-influencers for top ten proteins (named by their genes) in combined network of psychosis and antipsychotics sorted by Betweenness centrality.

In a previous study, we have compared the frequency of medication usage among $\mathrm{AD}+\mathrm{P}$ and $\mathrm{AD}-\mathrm{P}$ patients and conducted survival analysis on time to psychosis for AD patients to identify drugs with beneficial effects ${ }^{18}$. The results of our analysis revealed a significant association between Vitamin D use and delayed onset of psychotic symptoms. In addition, through the analysis of gene expression data, we found that AD- and/or psychosis-related genes were enriched in the list of genes most perturbed by Vitamin D. This observation provides us with a novel direction for the mechanism study of $\mathrm{AD}$ and psychosis, and may inspire the development of drugs to prevent or treat psychosis in $\mathrm{AD}$.

The role of Vitamin D in neurodegenerative diseases has been reported by many researchers. Six of the nine case-control studies found significant between-group differences illustrated by lower serum concentrations of 25-hydroxyvitamin $\mathrm{D}$, a metabolite of Vitamin $\mathrm{D}_{3}$, in $\mathrm{AD}$ cases compared to control groups ${ }^{19-25}$. Thus, Vitamin $\mathrm{D}$ Insufficiency is considered as a risk factor for $\mathrm{AD}$. However, Vitamin D's beneficial effect against $\mathrm{AD}+\mathrm{P}$ was freshly discovered and its mechanism may provide a unique viewpoint in preventing and treating $\mathrm{AD}+\mathrm{P}$.

Network approaches have been used in predicting and identifying the disease genes in multiple studies and some of the results have been verified ${ }^{26,27}$. It is suggested that, in the viewpoint of network biology, drug targets tend to locate at the transition area from the essential hubs, e.g. proteins interacting with more partner proteins, to redundant peripheral nodes ${ }^{28}$, e.g. proteins interacting with fewer partner proteins. The rationale behind this is a balance of toxicity and efficacy regarding the potential influence of the targets on cellular function.

The aim of this study is to explore potential molecular mechanisms that underlie the beneficial effects of Vitamin $\mathrm{D}$ on reducing psychosis symptoms in $\mathrm{AD}$ patients and to identify potential drug targets for $\mathrm{AD}+\mathrm{P}$ prevention or treatment by applying systems pharmacology approaches on analyzing their protein-protein interaction networks.

\section{Method and Material}

Gene dataset collection and pathway mapping. A network that includes both $\mathrm{AD}$ - and psychosis-related proteins were constructed and analyzed in order to study the crosstalk between them. AD- and psychosis-related genes were collected from multiple literatures and databases, including MetaCore from Clarivate Analytics (https://portal.genego.com/), GWAS Catalog for Genome Wide Association Studies (GWAS) (https:// www.ebi.ac.uk/GWAS/home) ${ }^{29}$ and BaseSpace Correlation Engine (https://www.illumina.com/index-d.html) ${ }^{30}$. These gene names were then converted to protein names by batch search function in the UniProt database. The criteria for including genes in our study are described in supplementary material. Vitamin D-perturbed genes and antipsychotics-perturbed genes were collected from BaseSpace Correlation Engine (https://www.illumina.com/ index-d.html $)^{30}$. Both down- and up-regulated genes were included into our analysis.

Signaling pathways for $\mathrm{AD}$ and psychosis were acquired from KEGG (http://www.genome.jp/kegg/) ${ }^{31}$ and PANTHER Classification System (http://pantherdb.org/) ${ }^{32}$.

Network analysis with centrality measures. In the following network analysis studies, we incorporated protein-protein interaction (PPI) data from STRING (https://string-db.org/) ${ }^{33}$ and the Online predicted human interaction database (OPHID) (http://ophid.utoronto.ca/ophidv2.204/index.jsp) ${ }^{34}$. The PPI network was constructed and analyzed with python package networkx (https://networkx.github.io/) ${ }^{35}$. The interaction 


\begin{tabular}{|l|l|l|l|l|}
\hline Network Name & $\begin{array}{l}\text { Node } \\
\text { Number }\end{array}$ & $\begin{array}{l}\text { Edge } \\
\text { Number }\end{array}$ & $\begin{array}{l}\text { Average Degree } \\
\text { Centrality }\end{array}$ & $\begin{array}{l}\text { Average Betweenness } \\
\text { Centrality }\end{array}$ \\
\hline AD & 1061 & 15691 & 0.0279 & 0.00167 \\
\hline Psychosis & 486 & 1409 & 0.0119 & 0.00642 \\
\hline AD-psychosis Combined Network & 1456 & 16989 & 0.0160 & 0.00158 \\
\hline
\end{tabular}

Table 3. Characteristics of AD- and Psychosis-related PPI networks.

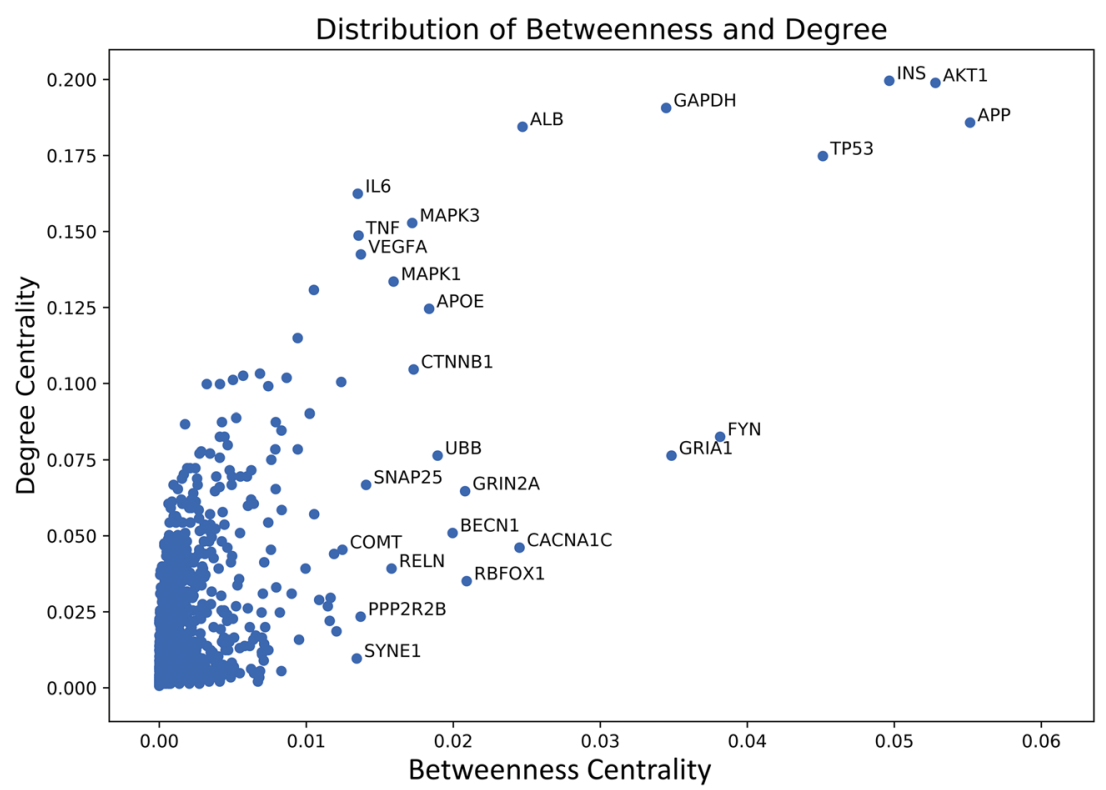

Figure 1. Distribution of Degree centrality and Betweenness centrality of nodes in the combined AD-psychosis PPI network. Most of the nodes have very low Degree centrality and Betweenness centrality while a very small group of nodes, like the top 10 nodes, possess very high centrality compared to others. This phenomenon suggests that the information flow within the network is controlled and regulated by the small group of nodes to a great extent.

network was shown in the molecular action view with the medium confidence level $(>0.4)^{36}$. The network containing $\mathrm{AD}$-related proteins (AD network) and the network containing psychosis-related proteins (Psychosis network) were joined to form a combined network (AD-psychosis combined network) for further study. PPI networks containing Vitamin D-perturbed proteins (Vitamin D network) and antipsychotics-perturbed proteins (Antipsychotics network) are also generated respectively.

Centrality measures of the nodes were introduced in network analysis to describe how the information will spread through the network. Two different kinds of centralities were included: Degree Centrality and Betweenness Centrality. Degree Centrality, as the most simple and direct, describes the number of connections of a particular node regardless of the direction and weight of the edges. Betweenness Centrality, as the centrality of control, represents the frequency at which a point occurs on the geodesic (shortest paths) that connect pairs of nodes. In another word, it quantifies how many times a particular node acts as a bridge linking two ends of the network.

Networks were processed and plotted with python package networkx ${ }^{35}$ and $\mathrm{Gephi}^{37}$. The centrality of nodes in the network was calculated based on the built-in algorithm of networkx ${ }^{35}$. In detail, the degree centrality values were normalized by dividing by the maximum possible degree in a simple graph $n-1$ where $n$ is the number of nodes in a network. The Betweenness centrality algorithm is from Ulkrik Brandes ${ }^{38-41}$.

In order to minimize the bias caused by the number of studies associated with different proteins, we use Betweenness centrality as our primary indicator in this study to learn more on the nodes' position in the network's structure, rather than the degree centrality of the nodes in the network.

Network analysis methods with centrality measures will first be examined with psychosis-related genes and known antipsychotics-perturbed targets. In order to do that, a combined network of psychosis network and antipsychotic network is constructed and the centrality measures are calculated as mentioned above. The connectivity parameters of known antipsychotic targets are examined to determine if they possess a significantly higher value.

To find sub-networks (communities) having different biological functions, community detection was further conducted in the combined network. The algorithm used for community detection was based on the Greedy Modularity Maximization method ${ }^{42,43}$. It begins with each node in its own community and joins the pair of communities that most increases modularity until no such pair exists. 


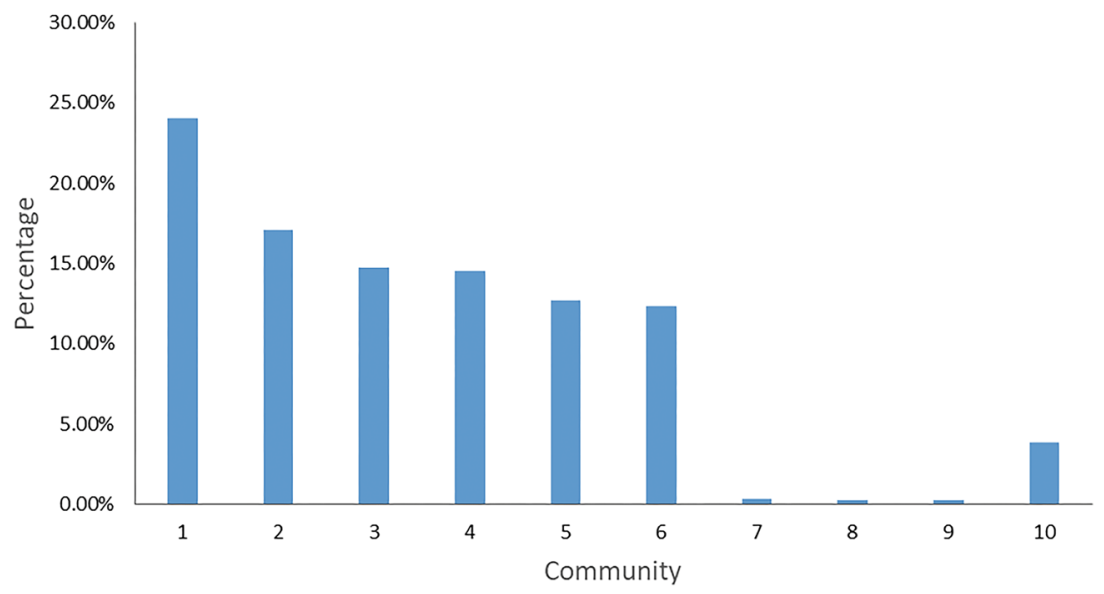

Figure 2. Overview of community detection. Seven meaningful communities are detected, and targets distributions are shown in the figure. These communities are constructed with similar targets amounts and can be the representatives for different biological functions involved.

Triple-focusing network approaches: identification of potential novel targets. Network analysis was further used to study a joint AD-psychosis-Vitamin D network in order to find potential drug targets for $\mathrm{AD}+\mathrm{P}$. The rationale of this approach was that the ideal potential targets should be in the overlapping part of PPI networks of $\mathrm{AD}$, psychosis and Vitamin $\mathrm{D}$ because the function of the potential targets can modulate the crosstalk between $\mathrm{AD}$ and psychosis and can also be regulated by Vitamin $\mathrm{D}$ through the Vitamin $\mathrm{D}$ receptor which is a transcriptional factor modulating gene expression. Thus, after constructing the AD-psychosis combined network and Vitamin D network, we overlapped them to explore the connectivity of these three parts and the roles of the triple-overlapped proteins.

Triple-focusing on AD-, psychosis-related and Vitamin D-perturbed proteins can help us reduce the artificial bias caused by the different amount of studies of those proteins, and also limit the potential side effects caused by targeting those very well-studied proteins which are usually located at the essential hubs. The identified small groups of proteins will have the potential to act as targets for Vitamin D to modulate AD- and psychosis-related networks.

\section{Results \\ Method verification with psychosis-related PPI network and antipsychotics-perturbed genes. Psychosis-related and antipsychotics-perturbed PPI networks are used to validate the network analy- sis methods we proposed. Characteristics of these two PPI networks and the combined network are shown below (Table 1). Five genes, DRD2, DRD3, HTR2A, OPRD1 and HTR7, are found shared by psychosis network and antipsychotics network. \\ The centrality measures of nodes in the psychosis and antipsychotics are calculated and the top ten nodes sorted by Betweenness values were shown in Table 2. As we expected, DRD2 and HTR2A, two major targets for current antipsychotics, were ranked as the first two proteins in our combined network when measured by Betweenness Centrality. If ranked by Degree centrality, ALB and FOS, two well-studied proteins, will have higher priority than HTR2A. The result revealed the great potential for proteins with a high Betweenness centrality being drug targets and provided a solid support for the method we proposed. Thus, the network analysis methods were applied to AD- and psychosis-related PPI networks.}

The AD-psychosis combined PPI network. In order to acquire a better understanding of the connection between $\mathrm{AD}$ and psychosis, and to further explore the potential drug targets suggested by the previous analysis, a combined PPI network of AD and psychosis was generated. One thousand and sixty-one AD-related genes and 15,691 PPIs of their protein products together with 483 psychosis-related genes and 1,361 psychosis-related PPIs were collected as the basis of our network. Among all the proteins collected, 90 proteins were shared by both AD and psychosis, including proteins encoded by SEMA3A, TUSC3, RPN2, AMBRA1, BECN1, CACNA1C, SGK1, ADAM10, GRIN2A, FYN, ANK3, TBXAS1, EFNA5, POLN, CHRNA3, NOTCH4, GRIA1, NTRK3, IQGAP2, RELN, NOS1, GPC6, TCF7L2, TCF4, MGLL, DRD3, CHRNA2, PAK2, CTNNA2, COL25A1, COL12A1, AGER, KIF26B, PPP2R2B, TEK, KALRN, PRKG1, KSR2, COLGALT2, MEIS1, SHISA9, ZKSCAN4, PTPRG, NKAPL, CTNNA3, PDE4B, HFE, MSR1, CSMD1, COMT, APBA1, IMMP2L, ELAVL4, LRRTM4, CDH13, ZNF804A, PBRM1, LRRN2, TEP1, STXBP5L, FHIT, SYNGAP1, ZSCAN31, TENM4, ABCB1, PLCL1, RBFOX1, FSTL5, SORCS3, NKAIN2, GLIS3, NXN, MAGI2, MEGF10, MPP6, TSPAN18, FRMD4B, MTHFD1L, TMTC1, LIN28B, UXS1, BICC1, ATXN7L1, EYS, GRAMD1B, TSPAN2, ENOX1, TMEM132D, CR1 and PCNX. The AD-psychosis combined network has 1,454 nodes and 16,948 PPIs. Characteristics of the combined network were most similar to those of $\mathrm{AD}$ network due to the disparity of the node numbers in $\mathrm{AD}$ - and psychosis-related PPI networks (Table 3). 


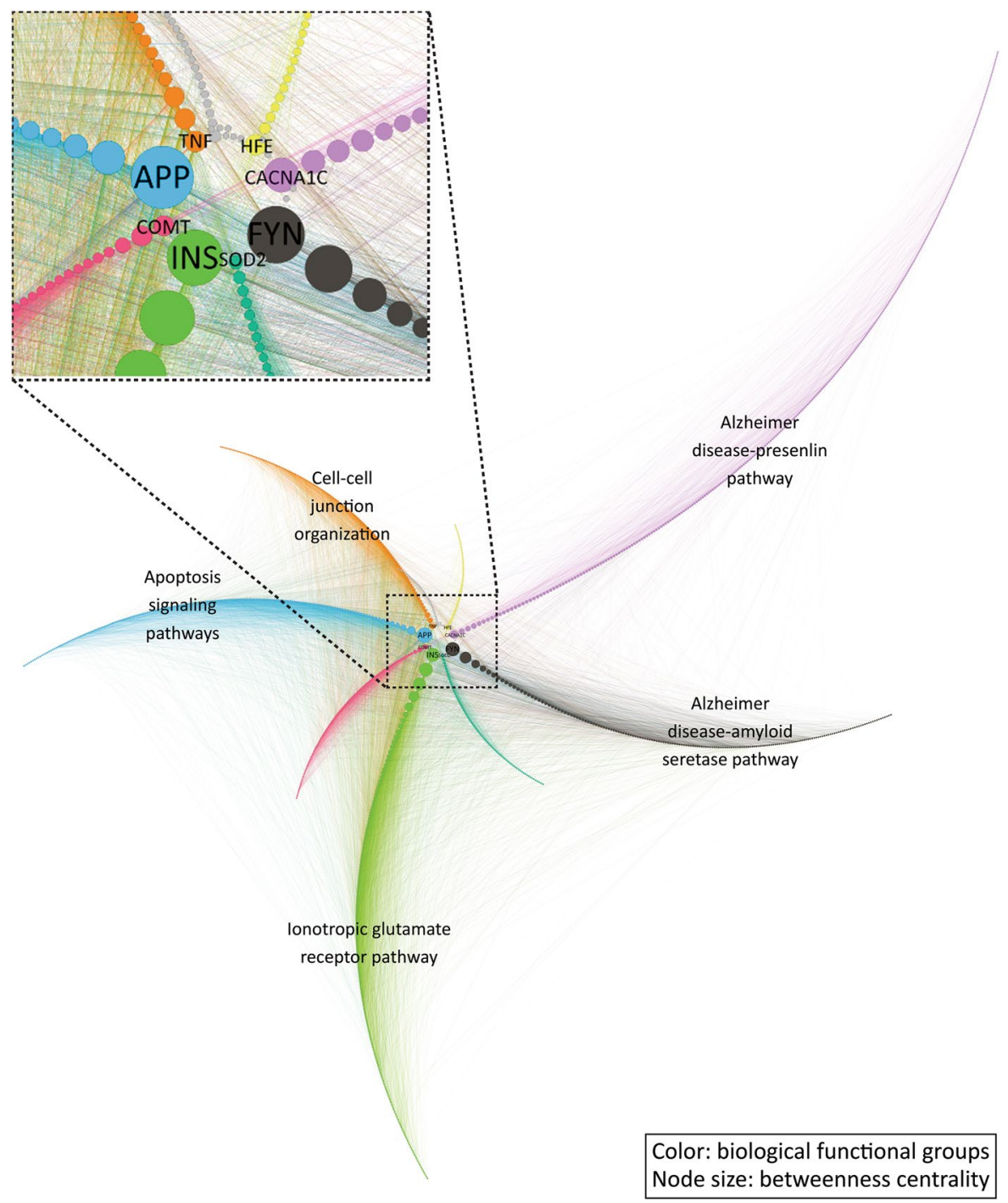

Figure 3. Overview of community interaction. Community interactions incorporated with the Betweenness centrality data of nodes and the functional annotations of the communities. The node size represents the Betweenness centrality of nodes. The high impact nodes, nodes with high Betweenness centrality, are evenly distributed to communities and function as the main gateway for information exchange and interactions. The architecture of the combined network is a big system formed by several sub-networks (communities) that connect with each other through a small hub, and most of the proteins in the network work mostly with the proteins within their communities. Figure generated with Gephi (https://gephi.org/) version 0.9.2.

\begin{tabular}{|l|l|}
\hline Gene (Degree Centrality) & Gene (Betweenness Centrality) \\
\hline INS $(0.200)$ & APP $(0.0552)$ \\
\hline AKT1 $(0.199)$ & AKT1 $(0.0528)$ \\
\hline GAPDH $(0.191)$ & INS $(0.0497)$ \\
\hline APP $(0.186)$ & TP53 $(0.0451)$ \\
\hline ALB $(0.184)$ & FYN $(0.0382)$ \\
\hline TP53(0.175) & GRIA1 $(0.0348)$ \\
\hline IL6(0.162) & GAPDH $(0.0345)$ \\
\hline MAPK3(0.153) & ALB $(0.0247)$ \\
\hline TNF $(0.149)$ & CACNA1C $(0.0245)$ \\
\hline VEGFA $(0.142)$ & RBFOX1 $(0.0209)$ \\
\hline
\end{tabular}

Table 4. Overview of top net-influencers in the AD-psychosis combined PPI network. 


\begin{tabular}{|l|l|l|}
\hline Community & Pathways (Pathway ID) & P-value \\
\hline Community 1 & FAS signaling pathway (P00020) & $<0.001$ \\
\hline Community 1 & Ras Pathway (P04393) & $<0.001$ \\
\hline Community 1 & PDGF signaling pathway (P00047) & $<0.001$ \\
\hline Community 1 & $\begin{array}{l}\text { Angiotensin II-stimulated signaling through G proteins and beta-arrestin } \\
\text { (P05911) }\end{array}$ & $<0.001$ \\
\hline Community 1 & Interleukin signaling pathway (P00036) & 0.00236 \\
\hline Community 1 & Wnt signaling pathway (P00057) & 0.00121 \\
\hline Community 1 & Huntington disease (P00029) & 0.00367 \\
\hline Community 1 & p53 pathway (P00059) & 0.00459 \\
\hline Community 1 & Alzheimer disease-presenilin pathway (P00004) & 0.00138 \\
\hline Community 1 & p38 MAPK pathway (P05918) & 0.0132 \\
\hline Community 1 & Parkinson disease (P00049) & 0.0135 \\
\hline Community 1 & Integrin signaling pathway (P00034) & 0.0294 \\
\hline Community 2 & Ionotropic glutamate receptor pathway (P00037) & $<0.001$ \\
\hline Community 2 & Muscarinic acetylcholine receptor 1 and 3 signaling pathway (P00042) & $<0.001$ \\
\hline Community 2 & 5HT1 type receptor-mediated signaling pathway (P04373) & $<0.001$ \\
\hline Community 2 & Enkephalin release (P05913) & $<0.001$ \\
\hline Community 2 & Synaptic vesicle trafficking (P05734) & $<0.001$ \\
\hline Community 2 & $\begin{array}{l}\text { Heterotrimeric G-protein signaling pathway-Gq alpha and Go alpha } \\
\text { mediated pathway (P00027) }\end{array}$ & $<0.001$ \\
\hline Community 2 & Metabotropic glutamate receptor group II pathway (P00040) & $<0.001$ \\
\hline Community 2 & Endothelin signaling pathway (P00019) & 0.00296 \\
\hline Community 2 & Opioid proopiomelanocortin pathway (P05917) & 0.00136 \\
\hline Community 3 & Alzheimer disease-amyloid secretase pathway (P00003) & $<0.001$ \\
\hline Community 4 & Apoptosis signaling pathway (P00006) & $<0.001$ \\
\hline Community 5 & Plasminogen activating cascade (P00050) & $<0.001$ \\
\hline Community 5 & Cholesterol biosynthesis (P00014) & 0.0223 \\
\hline Community 6 & Cadherin signaling pathway (P00012) & 0.0494 \\
\hline Community 10 & Cell-cell junction organization (R-HSA-421270) & 0.00992 \\
\hline Community 10 & Nectin/Necl trans heterodimerization (R-HSA-420597) & 0.0177 \\
\hline Community 10 & Cell junction organization (R-HSA-446728) & 0.0275 \\
\hline & & \\
\hline
\end{tabular}

Table 5. Results of protein-pathway mapping in the communities.

Top 10 net-influencers in the combined network are shown in Table 3 based on their Degree and Betweenness centralities respectively. It is not surprising that the 3 centralities overlapped with each other substantially, since they all measure the importance of the nodes in the whole network from different angles, and it is apparent that the top 10 nodes do have very higher values when compared with the average value, 10 -fold ratio at least. A better view is provided in Fig. 1 showing that only a few nodes take position at the upper-right corner. This phenomenon suggests that though there are 1,454 of nodes in the network, a small group of nodes, such as the top 10 nodes shown in the table, are extremely connected and play a critical role in the signaling process and information flow within the network.

After identifying the critical proteins in the network, the function of these proteins is our interest. We conducted pathway enrichment analysis to identify the underlying pathways participated by those proteins and therefore to establish a connection between proteins and their biological functions. Firstly, ten communities were detected as relatively separated components of the network (Fig. 2). Among the 10 detected communities, 7 communities, excluding 7, 8 and 9, contain enough nodes to be biologically meaningful. When sorting the network based on the community and the nodes' Betweenness, every community has one or a few nodes that possess a much higher Betweenness value and those nodes serve as the portal connecting the community to the other parts of the network (Fig. 3). Among the top 10 proteins we mentioned above (Table 4), APP, FYN, and INS are distributed into different communities as the leading nodes, which further illustrates the importance of these nodes in the combined network. These detected communities represent different biological pathways participating in the development of psychosis in AD. Secondly, protein-pathway mapping was conducted by comparing the proteins in the same community against the proteins in the pathways from online databases like KEGG.

The protein-pathway mapping returned a list of pathways associated with these 7 communities evidenced with very low False Discovery Rate (FDR) adjusted p-values, meaning that the proteins in these communities are highly accordant with proteins in these pathways recorded in the database. Pathways closely related to AD and neurological disorders (Table 5) were enriched in the list, including the Huntington disease pathway, Alzheimer's disease-presenilin pathway, p53 pathway and Alzheimer's disease-amyloid secretase pathway.

Figure 4 provided a more direct overview of the results of protein-pathway mapping. Community 1 and community 2 were mapped to multiple pathways with high credibility. It is fairly understandable because these two communities contain the largest amounts of targets and may result in mismatches. 


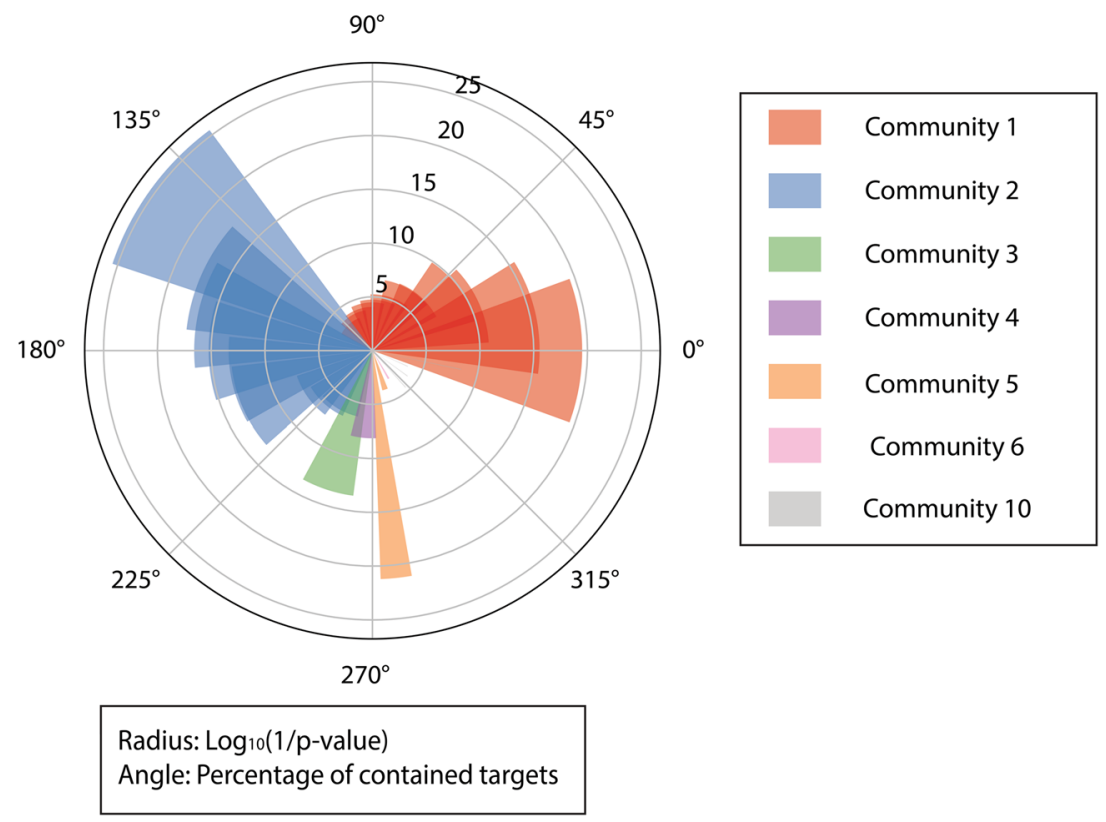

Figure 4. Distribution of proteins in the communities and p-values for protein-pathway mapping results. The radius represents the $\log _{10}(1 / \mathrm{p}$-value) of a mapping, and a higher bar has a smaller $\mathrm{p}$-value. The angle of the bar represents the percentage of proteins contained in the mapped community. Shades in same color indicate multiple pathway-matchings of one community. P-value is calculated by Fisher's exact test and all terms are adjusted by Benjamini-Hochberg FDR. Figure generated with matplotlib (https://matplotlib.org/) version $3.1 .3^{63}$.

Overlapping proteins between AD network and Psychosis network. Since the objective of this study is to study the development of psychosis in $\mathrm{AD}$, we focused on the overlapping proteins between $\mathrm{AD}$ and psychosis. The net-influence parameters of the 90 overlapped proteins are shown in Table 6. Most proteins in the overlapping part possess Betweenness values above average which further supports their bridging role in the networks.

Figure 5 shows the distribution of connectivity parameters of overlapping proteins. Even in the overlapping part of the network, the average Betweenness centrality remains relatively low and only a few nodes, like FYN and GRIA1, possess a much higher connectivity than other nodes. The distribution of Betweenness follows the same pattern as the whole network suggesting that even though 90 targets are found overlapped between psychosis and $\mathrm{AD}$, only a few of them are the "bridges" for the transferring of information.

Exploration of Vitamin D's beneficial effect through a triple-focusing approach. In our previously published paper, Vitamin D was identified as a promising medication with a significant association with decreased occurrences and delayed onset of $\mathrm{AD}+\mathrm{P}^{18}$. Therefore, we examined the relationship between the Vitamin D network and the AD-psychosis combined network. In total, 89 targets and 344 PPIs were collected in the Vitamin D network (Table 7). Among the 89 proteins, twenty-one are shared with the AD-psychosis combined network. Net influence parameters are calculated for these 21 targets and sorted by their Betweenness centrality values (Table 8 ).

After sorting by the Betweenness centrality, CACNA1C, COMT, NOTCH4 and DRD3 are ranked as the top four proteins. Their positions in the overlapping part of the combined network allow them to function more as a bridge to link different components of the network, which also suggests a therapeutic potential for $\mathrm{AD}+\mathrm{P}$. Therefore, these four proteins gained our special interest. One interesting thing is, when we look back at Fig. 1, these four targets fell into the middle distribution of values for Degree centrality and Betweenness centrality, which matched the conclusion that drug targets tend to be positioned at the transition area in a biological network ${ }^{28}$.

Figure 6 shows the distribution of connectivity parameters of overlapping proteins between AD-psychosis combined network and Vitamin D network. The 21 overlapped nodes followed the distribution of the whole combined network and revealed several proteins with outstanding Betweenness centrality values. These proteins will tend to act as the "bridges" in communicating AD-, psychosis-related network and Vitamin D perturbed network and thus the potential explanation of the beneficial effects of Vitamin $\mathrm{D}$ against $\mathrm{AD}+\mathrm{P}$.

\section{Discussion}

The network analysis based on the protein-protein interaction data have presented us four potential targets encoded by genes CACNA1C, NOTCH4, COMT and DRD3 that may account for the beneficial effects of Vitamin D against $\mathrm{AD}+\mathrm{P}$. These four potential targets all possess high enough connectivity to alter the crosstalk between $\mathrm{AD}$ and psychosis. In addition, variants in $C A C N A 1 C, N O T C H 4$ and $C O M T$ had been reported to be associated with schizophrenia in GWAS studies ${ }^{4-46}$. Among them, the function of CACNA1C, NOTCH4 and COMT were reported to be closely associated with calcium homeostasis ${ }^{47-53}$ which can be further associated with Vitamin D’s effect. Similarly, after the 


\begin{tabular}{|c|c|c|}
\hline Gene Name & Degree Centrality & Betweenness Centrality \\
\hline SEMA3A & 0.0220 & 0.0046 \\
\hline TUSC3 & 0.0048 & 0.0025 \\
\hline RPN2 & 0.0048 & 0.0019 \\
\hline AMBRA1 & 0.0055 & 0.0002 \\
\hline BECN1 & 0.0509 & 0.020 \\
\hline CACNA1C & 0.0461 & 0.0245 \\
\hline SGK1 & 0.033 & 0.008 \\
\hline ADAM10 & 0.0571 & 0.0105 \\
\hline GRIN2A & 0.0647 & 0.0208 \\
\hline FYN & 0.0826 & 0.0382 \\
\hline ANK3 & 0.0268 & 0.0115 \\
\hline TBXAS1 & 0.0083 & 0.0021 \\
\hline EFNA5 & 0.0255 & 0.0042 \\
\hline POLN & 0.0055 & 0.0026 \\
\hline CHRNA3 & 0.0117 & 0.0012 \\
\hline NOTCH4 & 0.020 & 0.0072 \\
\hline GRIA1 & 0.0764 & 0.0348 \\
\hline NTRK3 & 0.0248 & 0.007 \\
\hline IQGAP2 & 0.0055 & 0.0038 \\
\hline RELN & 0.0392 & 0.0158 \\
\hline NOS1 & 0.044 & 0.0119 \\
\hline GPC6 & 0.0145 & 0.0071 \\
\hline TCF7L2 & 0.0296 & 0.0117 \\
\hline TCF4 & 0.020 & 0.0062 \\
\hline MGLL & 0.0172 & 0.0066 \\
\hline DRD3 & 0.0482 & 0.0043 \\
\hline CHRNA2 & 0.0145 & 0.0007 \\
\hline PAK2 & 0.0241 & 0.0046 \\
\hline CTNNA2 & 0.022 & 0.0116 \\
\hline COL25A1 & 0.0124 & 0.0035 \\
\hline COL12A1 & 0.011 & 0.0015 \\
\hline AGER & 0.0303 & 0.0042 \\
\hline KIF26B & 0.0055 & \begin{tabular}{|l|}
0.0007 \\
\end{tabular} \\
\hline PPP2R2B & 0.0234 & 0.0137 \\
\hline TEK & 0.0262 & 0.0060 \\
\hline KALRN & 0.0289 & 0.0109 \\
\hline PRKG1 & 0.0310 & 0.0070 \\
\hline KSR2 & 0.0103 & 0.0022 \\
\hline COLGALT2 & 0.0076 & 0.0009 \\
\hline MEIS1 & 0.0117 & 0.0020 \\
\hline SHISA9 & 0.0096 & 0.0006 \\
\hline ZKSCAN4 & 0.0055 & 0.0069 \\
\hline PTPRG & 0.0151 & 0.0021 \\
\hline NKAPL & 0.0055 & 0.0043 \\
\hline CTNNA3 & 0.0124 & 0.0024 \\
\hline PDE4B & 0.02 & 0.0037 \\
\hline HFE & 0.0186 & 0.0121 \\
\hline MSR1 & 0.0248 & 0.0082 \\
\hline CSMD1 & 0.0138 & 0.0058 \\
\hline COMT & 0.0454 & 0.0125 \\
\hline APBA1 & 0.0248 & 0.0044 \\
\hline IMMP2L & 0.0124 & 0.0047 \\
\hline ELAVL4 & 0.0165 & 0.0051 \\
\hline LRRTM4 & 0.0062 & 0.0006 \\
\hline CDH13 & 0.0110 & 0.0023 \\
\hline ZNF804A & 0.0151 & \begin{tabular}{|l|}
0.0048 \\
\end{tabular} \\
\hline PBRM1 & 0.0096 & 0.0026 \\
\hline \multicolumn{3}{|l|}{ Continued } \\
\hline
\end{tabular}




\begin{tabular}{|l|l|l|}
\hline Gene Name & Degree Centrality & Betweenness Centrality \\
\hline LRRN2 & 0.0028 & 0.0009 \\
\hline TEP1 & 0.0062 & 0.0050 \\
\hline STXBP5 & 0.0124 & 0.0074 \\
\hline FHIT & 0.0165 & 0.0044 \\
\hline SYNGAP1 & 0.0193 & 0.0013 \\
\hline ZSCAN31 & 0.0034 & 0.0003 \\
\hline TENM4 & 0.0076 & 0.0017 \\
\hline ABCB1 & 0.0310 & 0.009 \\
\hline PLCL1 & 0.0028 & 0.0002 \\
\hline RBFOX1 & 0.0351 & 0.0209 \\
\hline FSTL5 & 0.0048 & 0.0019 \\
\hline SORCS3 & 0.0055 & 0.0045 \\
\hline NKAIN2 & 0.0041 & 0.0003 \\
\hline GLIS3 & 0.0069 & 0.0031 \\
\hline NXN & 0.0083 & 0.0017 \\
\hline MAGI2 & 0.0145 & 0.0044 \\
\hline MEGF10 & 0.0034 & 0.0003 \\
\hline MPP6 & 0.0055 & 0.0003 \\
\hline TSPAN18 & 0.0028 & 0.0004 \\
\hline FRMD4B & 0.0021 & 0.0002 \\
\hline MTHFD1L & 0.0103 & 0.0006 \\
\hline TMTC1 & 0.0034 & 0.0001 \\
\hline LIN28B & 0.0034 & 0.0012 \\
\hline UXS1 & 0.0048 & 0.0064 \\
\hline BICC1 & 0.0055 & 0.0083 \\
\hline ATXN7L1 & 0.0048 & 0.0019 \\
\hline EYS & 0.0069 & 0.0024 \\
\hline GRAMD1B & 0.0028 & 0.0027 \\
\hline TSPAN2 & 0.0048 & 0.0018 \\
\hline ENOX1 & 0.0014 & 0 \\
\hline TMEM132D & 0.0048 & 0.0055 \\
\hline CR1 & 0.0124 & 0.0004 \\
\hline PCNX & 0.0014 & 0.0001 \\
\hline & & \\
\hline & & \\
\hline
\end{tabular}

Table 6. Overview of net-influencers for overlapping proteins (named by their genes) between AD network and Psychosis network.

activation of DRD3 by dopamine, the G $\beta \gamma$ complex is released and can interact directly with voltage-gated calcium channels $s^{54,55}$. Except for NOTCH4, all are targeted by marketed drugs for different indications. Interestingly, DRD3 is one of the primary targets for antipsychotics in treating psychotic symptoms in schizophrenia or other neurological disorders ${ }^{56,57}$. Alternative splicing of DRD3 in the transcription process may result in encoding different isoforms that are functionally impaired ${ }^{58}$. Although limited, there is some support for targeting DRD3 in the treatment of $A D+\mathrm{P}^{59,60}$. However, verification of DRD3 or the other of these four potential targets for $\mathrm{AD}+\mathrm{P}$ will require additional studies.

The beneficial effect of Vitamin D against AD have been widely reported. The protective effect of Vitamin D can be executed by reducing the oxidative and nitrosative damage caused by elevated levels of nitric oxide (NO) and inducible nitric oxide synthase (iNOS) in nerve cells ${ }^{61}$. There is also evidence suggesting an overlap between the disruptions of vitamin D pathways with amyloid pathology which can partially explain the protective role of Vitamin $\mathrm{D}$ in $\mathrm{AD}^{62}$. However, this study is the first study to explore the mechanism of Vitamin D's beneficial effect against $\mathrm{AD}+\mathrm{P}$. In this study, the triple-focusing approach we use can help minimize the bias caused amount of studies and restrain our scope at Vitamin D related potential targets.

There are limitations in this study. The PPI networks were constructed based on the protein-protein interaction data extracted from databases, thus they are limited by the amount and availability of data in the databases. Also, there is no direction information attached with most PPIs which means our PPI networks are undirected. Therefore, centrality measures can be biased by the direction information in actual situations.

\section{Conclusion}

In this study, various approaches of network analysis are incorporated with systems pharmacology to provide a systematic overview on the crosstalk among $\mathrm{AD}$, psychosis and Vitamin $\mathrm{D}$ at the molecular level. The triple-focusing network method helps us explore the designated mechanisms for Vitamin D's effects on $\mathrm{AD}+\mathrm{P}$ and a potential explanation is provided: Vitamin $\mathrm{D}$ regulates several genes encoding proteins that play critical 


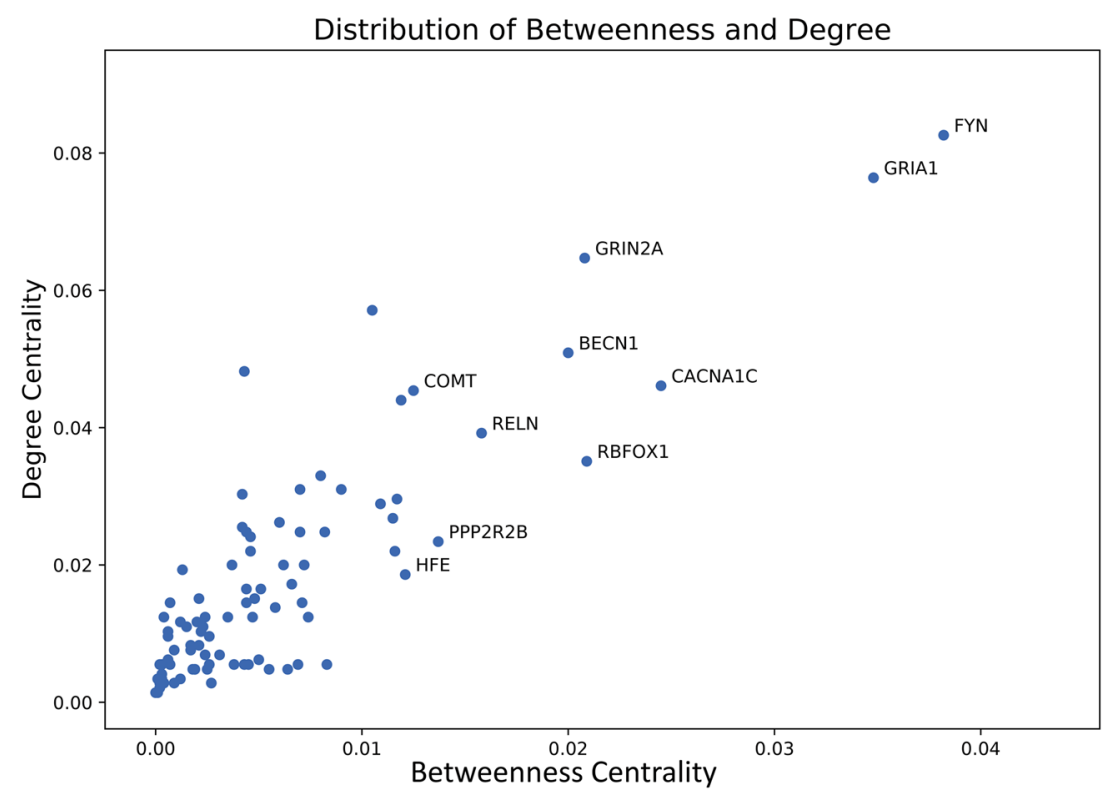

Figure 5. Distribution of Degree centrality and Betweenness centrality of overlapping proteins between AD network and psychosis network. FYN and GRIA1, as members of the top 10 targets, possess a far larger Degree centrality and Betweenness centrality among the overlapping proteins. Figure generated with matplotlib (https://matplotlib.org/) version 3.1.3 ${ }^{63}$.

\begin{tabular}{|l|l|l|l|l|}
\hline $\begin{array}{l}\text { Network } \\
\text { Name }\end{array}$ & $\begin{array}{l}\text { Node } \\
\text { Number }\end{array}$ & $\begin{array}{l}\text { Edge } \\
\text { Number }\end{array}$ & $\begin{array}{l}\text { Average Degree } \\
\text { Centrality }\end{array}$ & $\begin{array}{l}\text { Average Betweenness } \\
\text { Centrality }\end{array}$ \\
\hline Vitamin D & 89 & 344 & 0.0869 & 0.018 \\
\hline
\end{tabular}

Table 7. Characteristics of Vitamin D network.

\begin{tabular}{|l|l|l|}
\hline Gene Name & Degree Centrality & Betweenness Centrality \\
\hline CACNA1C & 0.0461 & 0.0245 \\
\hline COMT & 0.0454 & 0.0125 \\
\hline NOTCH4 & 0.02 & 0.0072 \\
\hline DRD3 & 0.0482 & 0.0043 \\
\hline CD36 & 0.022 & 0.0024 \\
\hline EGR1 & 0.0619 & 0.0022 \\
\hline CCL2 & 0.0867 & 0.0018 \\
\hline DLX5 & 0.0062 & 0.0010 \\
\hline CYP1A1 & 0.0227 & 0.0008 \\
\hline A2M & 0.0358 & 0.0006 \\
\hline VDR & 0.0282 & 0.0006 \\
\hline TGFB2 & 0.0296 & 0.0006 \\
\hline TIMP3 & 0.0268 & 0.0006 \\
\hline CD14 & 0.0227 & 0.0006 \\
\hline CYP19A1 & 0.0296 & 0.0004 \\
\hline NME1 & 0.0227 & 0.0003 \\
\hline HSD11B1 & 0.0131 & 0.0002 \\
\hline MMP12 & 0.0227 & 0.0002 \\
\hline AMBRA1 & 0.0055 & 0.0002 \\
\hline ALOX15 & 0.0117 & 0.0001 \\
\hline GIG25 & 0.0145 & 0.0001 \\
\hline & & \\
\hline
\end{tabular}

Table 8. Overview of top net-influencers ranked by Betweenness values for overlapping proteins (named by their genes) between AD-psychosis combined network and Vitamin D network. 


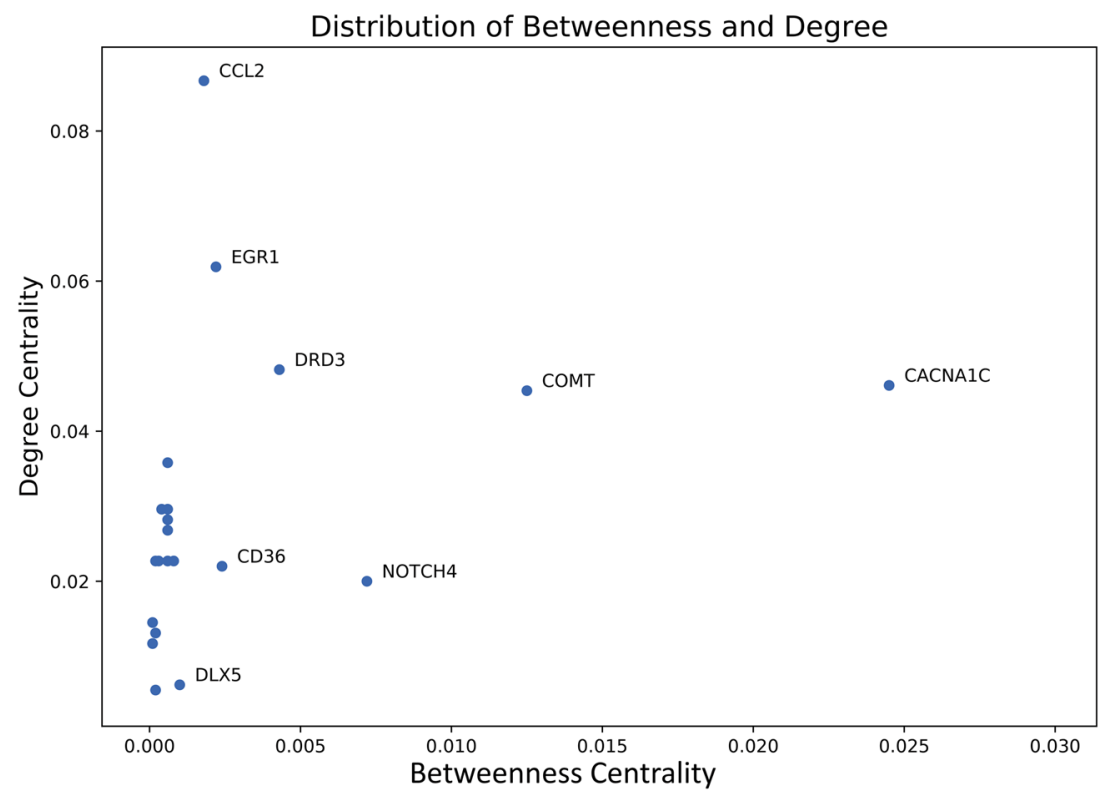

Figure 6. Distribution of Degree centrality and Betweenness centrality of overlapping proteins between ADpsychosis combined network and Vitamin D network. Overlapping proteins between AD-psychosis combined network and Vitamin D network follows the same pattern as the whole networks. Some nodes like CACNA1C, COMT, NOTCH4 and DRD3 possess much higher Betweenness centrality values than the average value of the network. Figure generated with matplotlib (https://matplotlib.org/) version 3.1.363.

roles in the overlapping part of the AD-psychosis combined network, which allow them maximally influence the signaling and information transfer process. In other words, proteins with high net-influence that localize at the triple-overlapped part of the AD, psychosis and Vitamin D network, like CACNA1C, COMT, NOTCH4 and $D R D 3$, possess the ability to play an important role in the crosstalk among $\mathrm{AD}$ and psychosis by delivering Vitamin D's effect to the transiting hub connecting the AD network and psychosis network. Thus, the four identified potential targets can be crucial in explaining Vitamin D's beneficial effect against AD $+\mathrm{P}$. To conclude, the results from this study provided a possible explanation of the beneficial effect of Vitamin $\mathrm{D}$ against $\mathrm{AD}+\mathrm{P}$ and presented a new direction for drug development with four potential novel targets.

\section{Data Availability}

Full gene lists used in this study can be found in the supplementary material. The detailed PPI interaction data and full net-influence parameters list are available on request.

Received: 23 October 2019; Accepted: 21 March 2020;

Published online: 09 April 2020

\section{References}

1. Thorgrimsen, L. et al. Whose quality of life is it anyway?: The validity and reliability of the Quality of Life-Alzheimer's Disease (QoLAD) scale. Alzheimer Disease \& Associated Disorders 17, 201-208 (2003).

2. Wimo, A. \& Prince, M. World Alzheimer Report. The global economic impact of dementia. Alzheimer's Disease International (2010).

3. Blacker, D. et al. ApoE-4 and age at onset of Alzheimer's disease: the NIMH genetics initiative. Neurology 48, 139-147 (1997).

4. Killin, L. O. J., Starr, J. M., Shiue, I. J. \& Russ, T. C. Environmental risk factors for dementia: a systematic review. BMC Geriatr 16, 175-175, https://doi.org/10.1186/s12877-016-0342-y (2016).

5. Murray, P. S., Kumar, S., Demichele-Sweet, M. A. \& Sweet, R. A. Psychosis in Alzheimer's disease. Biological psychiatry 75, 542-552, https://doi.org/10.1016/j.biopsych.2013.08.020 (2014).

6. Ropacki, S. A. \& Jeste, D. V. Epidemiology of and risk factors for psychosis of Alzheimer's disease: a review of 55 studies published from 1990 to 2003. The American journal of psychiatry 162, 2022-2030, https://doi.org/10.1176/appi.ajp.162.11.2022 (2005).

7. Gilley, D. W., Wilson, R. S., Beckett, L. A. \& Evans, D. A. Psychotic symptoms and physically aggressive behavior in Alzheimer's disease. Journal of the American Geriatrics Society 45, 1074-1079 (1997).

8. Sweet, R. A. et al. The 5-HTTPR polymorphism confers liability to a combined phenotype of psychotic and aggressive behavior in Alzheimer disease. International psychogeriatrics 13, 401-409 (2001).

9. Lyketsos, C. G. et al. Neuropsychiatric disturbance in Alzheimer's disease clusters into three groups: the Cache County study. International journal of geriatric psychiatry 16, 1043-1053 (2001).

10. Sweet, R. A., Bennett, D. A., Graff-Radford, N. R. \& Mayeux, R. Assessment and familial aggregation of psychosis in Alzheimer's disease from the National Institute on Aging Late Onset Alzheimer's Disease Family Study. Brain: a journal of neurology 133, 1155-1162, https://doi.org/10.1093/brain/awq001 (2010).

11. Wilson, R. S. et al. Hallucinations, cognitive decline, and death in Alzheimer's disease. Neuroepidemiology 26, 68-75, https://doi. org/10.1159/000090251 (2006).

12. Scarmeas, N. et al. Delusions and hallucinations are associated with worse outcome in Alzheimer disease. Archives of neurology 62, 1601-1608, https://doi.org/10.1001/archneur.62.10.1601 (2005).

13. Kaufer, D. I. et al. Assessing the impact of neuropsychiatric symptoms in Alzheimer's disease: the Neuropsychiatric Inventory Caregiver Distress Scale. Journal of the American Geriatrics Society 46, 210-215 (1998). 
14. Garety, P. \& Freeman, D. The past and future of delusions research: from the inexplicable to the treatable. The British Journal of Psychiatry 203, 327-333 (2013).

15. Eastvold, A., Heaton, R. \& Cadenhead, K. Neurocognitive deficits in the (putative) prodrome and first episode of psychosis. Schizophrenia research 93, 266-277 (2007).

16. Link, B. G., Monahan, J., Stueve, A. \& Cullen, F. T. Real in their consequences: A sociological approach to understanding the association between psychotic symptoms and violence. American Sociological Review, 316-332 (1999).

17. Gilley, D. W., Whalen, M. E., Wilson, R. S. \& Bennett, D. A. Hallucinations and associated factors in Alzheimer's disease. The Journal of neuropsychiatry and clinical neurosciences (1991).

18. Wang, L. et al. Effects of Vitamin D Use on Outcomes of Psychotic Symptoms in Alzheimer's disease Patients. The American Journal of Geriatric Psychiatry (2019).

19. Martyn, C., Singh, S. \& Wood, P. Calcium metabolism in Alzheimer’s disease. Gerontology 35, 153-157 (1989).

20. FERRIER, I. N. et al. Reduced gastrointestinal absorption of calcium in dementia. Age and ageing 19, 368-375 (1990).

21. Kipen, E., Helme, R. D., Wark, J. D. \& Flicker, L. Bone density, vitamin D nutrition, and parathyroid hormone levels in women with dementia. Journal of the American Geriatrics Society 43, 1088-1091 (1995).

22. Sato, Y. et al. Vitamin K deficiency and osteopenia in elderly women with Alzheimer's disease. Archives of physical medicine and rehabilitation 86, 576-581 (2005).

23. Evatt, M. L. et al. Prevalence of vitamin D insufficiency in patients with Parkinson disease and Alzheimer disease. Archives of neurology 65, 1348-1352 (2008).

24. Luckhaus, C. et al. Blood biomarkers of osteoporosis in mild cognitive impairment and Alzheimer's disease. Journal of neural transmission 116, 905-911 (2009).

25. Buell, J. et al. 25-Hydroxyvitamin D, dementia, and cerebrovascular pathology in elders receiving home services. Neurology 74, $18-26(2010)$.

26. Oti, M., Snel, B., Huynen, M. A. \& Brunner, H. G. Predicting disease genes using protein-protein interactions. Journal of medical genetics 43, 691-698 (2006).

27. Krauthammer, M., Kaufmann, C. A., Gilliam, T. C. \& Rzhetsky, A. Molecular triangulation: bridging linkage and molecular-network information for identifying candidate genes in Alzheimer's disease. Proceedings of the National Academy of Sciences 101, 15148-15153 (2004).

28. Hopkins, A. L. Network pharmacology. Nature Biotechnology 25, 1110, https://doi.org/10.1038/nbt1007-1110 (2007).

29. Buniello, A. et al. The NHGRI-EBI GWAS Catalog of published genome-wide association studies, targeted arrays and summary statistics 2019. Nucleic acids research 47, D1005-D1012 (2018).

30. Eberle, M. A. et al. A reference data set of 5.4 million phased human variants validated by genetic inheritance from sequencing a three-generation 17-member pedigree. Genome research 27, 157-164 (2017).

31. Kanehisa, M. \& Goto, S. KEGG: kyoto encyclopedia of genes and genomes. Nucleic Acids Res 28, 27-30 (2000).

32. Mi, H., Muruganujan, A., Casagrande, J. T. \& Thomas, P. D. Large-scale gene function analysis with the PANTHER classification system. Nature Protocols 8, 1551, 10.1038/nprot.2013.092 https://www.nature.com/articles/nprot.2013.092\#supplementaryinformation (2013).

33. Jensen, L. J. et al. STRING 8-a global view on proteins and their functional interactions in 630 organisms. Nucleic Acids Res 37, D412-416, https://doi.org/10.1093/nar/gkn760 (2009)

34. Brown, K. R. \& Jurisica, I. Online predicted human interaction database. Bioinformatics (Oxford, England) 21, 2076-2082, https:// doi.org/10.1093/bioinformatics/bti273 (2005).

35. Exploring Network Structure, Dynamics, and Function using NetworkX (Proceedings of the 7th Python in Science Conference, 2008).

36. Franceschini, A. et al. STRING v9. 1: protein-protein interaction networks, with increased coverage and integration. Nucleic acids research 41, D808-D815 (2012).

37. Bastian, M., Heymann, S. \& Jacomy, M. In Third international AAAI conference on weblogs and social media.

38. Brandes, U. A faster algorithm for betweenness centrality. Journal of mathematical sociology 25, 163-177 (2001).

39. Brandes, U. On variants of shortest-path betweenness centrality and their generic computation. Social Networks 30, 136-145 (2008).

40. Brandes, U. \& Pich, C. Centrality estimation in large networks. International Journal of Bifurcation and Chaos 17, 2303-2318 (2007).

41. Freeman, L. C. A set of measures of centrality based on betweenness. Sociometry, 35-41 (1977).

42. Newman, M. E. Communities, modules and large-scale structure in networks. Nature physics 8, 25 (2012).

43. Clauset, A., Newman, M. E. \& Moore, C. Finding community structure in very large networks. Physical review E 70, 066111 (2004).

44. Ripke, S. et al. Biological insights from 108 schizophrenia-associated genetic loci. Nature 511, 421 (2014).

45. Stefansson, H. et al. Common variants conferring risk of schizophrenia. Nature 460, 744 (2009).

46. Goes, F. S. et al. Genome-wide association study of schizophrenia in Ashkenazi Jews. American Journal of Medical Genetics Part B: Neuropsychiatric Genetics 168, 649-659 (2015).

47. Consortium, T. U. UniProt: a worldwide hub of protein knowledge. Nucleic Acids Research 47, D506-D515, https://doi.org/10.1093/ nar/gky1049 (2018).

48. Rand, M. D. et al. Calcium depletion dissociates and activates heterodimeric notch receptors. Molecular and cellular biology 20 , 1825-1835 (2000).

49. Yang, H., Ahn, C. \& Jeung, E.-B. Differential expression of calcium transport genes caused by COMT inhibition in the duodenum, kidney and placenta of pregnant mice. Molecular and Cellular Endocrinology 401, 45-55, https://doi.org/10.1016/j.mce.2014.11.020 (2015).

50. Horwitz, M. J., Hodak, S. P. \& Stewart, A. F. Non-parathyroid hypercalcemia. Primer on the Metabolic Bone Diseases and Disorders of Mineral Metabolism, 7th edn. American Society for Bone and Mineral Research: Washington, DC, 307-312 (2009).

51. Silverberg, S. J. \& Bilezikian, J. P. Primary hyperparathyroidism: still evolving? Journal of Bone and Mineral Research 12, 856-862 (1997).

52. Quiram, D. \& Weinshilboum, R. M. Catechol-o-methyltransferase in rat erythrocyte and three other tissues: comparison of biochemical properties after removal of inhibitory calcium 1. Journal of neurochemistry 27, 1197-1203 (1976).

53. Papaleo, F. et al. Genetic dissection of the role of catechol-O-methyltransferase in cognition and stress reactivity in mice. Journal of Neuroscience 28, 8709-8723 (2008).

54. Ustione, A. \& Piston, D. W. Dopamine synthesis and D3 receptor activation in pancreatic $\beta$-cells regulates insulin secretion and intracellular [Ca2+] oscillations. Molecular endocrinology 26, 1928-1940 (2012).

55. Caldwell, B., Ustione, A. \& Piston, D. Fluorescence fluctuation spectroscopy to detect interactions between dopamine receptors and calcium channel in pancreatic $\beta$-cells. Biophysical Journal 106, 718a (2014).

56. Joyce, J. N. \& Millan, M. J. Dopamine D3 receptor agonists for protection and repair in Parkinson's disease. Current opinion in pharmacology 7, 100-105, https://doi.org/10.1016/j.coph.2006.11.004 (2007).

57. Benedetti, F. et al. Dopamine receptor D2 and D3 gene variants are not associated with the antidepressant effect of total sleep deprivation in bipolar depression. Psychiatry research 118, 241-247 (2003).

58. Imbriano, C. \& Molinari, S. Alternative Splicing of Transcription Factors Genes in Muscle Physiology and Pathology. Genes (Basel) 9, 107, https://doi.org/10.3390/genes9020107 (2018) 
59. Sweet, R. A. et al. Alterations of striatal dopamine receptor binding in Alzheimer disease are associated with Lewy body pathology and antemortem psychosis. Archives of neurology 58, 466-472, https://doi.org/10.1001/archneur.58.3.466 (2001).

60. Reeves, S. et al. Therapeutic window of dopamine D2/3 receptor occupancy to treat psychosis in Alzheimer's disease. Brain: a journal of neurology 140, 1117-1127, https://doi.org/10.1093/brain/aww359 (2017).

61. Dursun, E., Gezen-Ak, D. \& Yilmazer, S. A new mechanism for amyloid- $\beta$ induction of iNOS: vitamin D-VDR pathway disruption. Journal of Alzheimer's Disease 36, 459-474 (2013).

62. Gezen-Ak, D., Yllmazer, S. \& Dursun, E. Why vitamin D in Alzheimer's disease? The hypothesis. Journal of Alzheimer's Disease 40, 257-269 (2014).

63. Hunter, J. D. Matplotlib: A 2D Graphics Environment. Computing in Science \& Engineering 9, 90-95, https://doi.org/10.1109/ MCSE.2007.55 (2007).

\section{Acknowledgements}

Authors would like to acknowledge the funding supports by National Institutes of Health grants MH116046, AG027224 and AG005133.

\section{Author contributions}

P.F., L.W. and R.S designed the experiments, P.F. conducted the experiments, analyzed the results and prepared figures. P.F., X.Q. and L.W. contributed to scientific discussions and data interpretations. R.S. provided scientific directions and consulting. P.F., L.W. and R.S wrote the manuscript. All authors reviewed the manuscript.

\section{Competing interests}

The authors declare no competing interests.

\section{Additional information}

Supplementary information is available for this paper at https://doi.org/10.1038/s41598-020-63021-8.

Correspondence and requests for materials should be addressed to R.A.S. or L.W.

Reprints and permissions information is available at www.nature.com/reprints.

Publisher's note Springer Nature remains neutral with regard to jurisdictional claims in published maps and institutional affiliations.

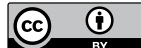

Open Access This article is licensed under a Creative Commons Attribution 4.0 International License, which permits use, sharing, adaptation, distribution and reproduction in any medium or format, as long as you give appropriate credit to the original author(s) and the source, provide a link to the Creative Commons license, and indicate if changes were made. The images or other third party material in this article are included in the article's Creative Commons license, unless indicated otherwise in a credit line to the material. If material is not included in the article's Creative Commons license and your intended use is not permitted by statutory regulation or exceeds the permitted use, you will need to obtain permission directly from the copyright holder. To view a copy of this license, visit http://creativecommons.org/licenses/by/4.0/.

(C) The Author(s) 2020 